Annuaire suisse de politique de développement

4 | 1984

Annuaire Suisse - Tiers Monde 1984

\title{
3. Aide publique au développement
}

\section{Antoine Brawand}

\section{OpenEdition}

\section{Journals}

Édition électronique

URL : http://journals.openedition.org/aspd/1175

DOI : $10.4000 /$ aspd. 1175

ISSN : 1663-9669

\section{Éditeur}

Institut de hautes études internationales et du développement

\section{Édition imprimée}

Date de publication : 1 janvier 1984

Pagination : 299-316

ISSN : 1660-5934

\section{Référence électronique}

Antoine Brawand, «3. Aide publique au développement», Annuaire suisse de politique de développement [En ligne], 4 | 1984, mis en ligne le 03 février 2013, consulté le 08 septembre 2020. URL : http:// journals.openedition.org/aspd/1175; DOI : https://doi.org/10.4000/aspd.1175 


\section{TRAITS MARQUANTS}

Pour situer l'aide publique au développement dans le contexte général des flux financiers déclarés par les pays membres du Comité d'aide au développement (CAD) de I'OCDE, voir les tableaux 2.1. et 2.2. (section précédente).

\section{a) Aperçu général de l'aide publique au développement (APD) (Tableaux 3.1.)}

Plus des deux tiers des prestations suisses consistent en versements bilatéraux (de pays à pays) (2). Le reste correspond aux contributions générales de la Suisse à des institutions internationales de développement et d'aide humanitaire (voir tableau 3.5.). La part des dons est élevée : en moyenne $88 \%$ de I'aide accordée au cours des trois dernières années (sans compter les versements faits aux fonds régionaux de développement, qui représentent en réalité aussi des dons).

Les principaux types de prestations de la Suisse sont la coopération technique (près de la moitié des versements nets) et l'aide financière (près d'un cinquième). Les prestations sont essentiellement assurées par la Confédération. Les versements (volontaires) des cantons et des communes s'élèvent à près d'un et demi pourcent de l'aide publique suisse.

\section{b) Répartition géographique de l'aide bilatérale (Tableaux 3.2.)}

Les principales destinations de l'aide bilatérale suisse sont l'Afrique (plus des deux cinquièmes en 1983) et l'Asie (plus d'un quart). L'Amérique latine reçoit nettement moins (un septième en 1983). La répartition par continents correspond à peu près à celle de l'aide accordée par l'ensemble des pays mem.

1. Un tableau (3.6.) montre les dons des institutions privées bénévoles suisses. Ces prestations correspondent à une catégorie de flux financiers distincts de l'aide publique au développement (voir tableaux 2.1., dans la section précédente). Elles sont insérées ici pour simplifier la présentation des statistiques.

2. Depuis 1979 , les contributions versées aux institutions internationales pour des projets de développement spécifiques sont comptées dans l'aide bilatérale. En 1983, ce type de prestations (aide associée, cofinancements et contributions à des instituts de recherche) s'est élevé à $12 \%$ de l'aide publique suisse au développement. (Voir tableau 3.3.D.) 
bres du CAD. Notons cependant que la Suisse accorde une priorité plus nette à I'Afrique. La part de I'Asie est légèrement plus faible et celle de I'Océanie est presque nulle.

La répartition par pays diffère en revanche fondamentalement (voir le classement des vingt premiers destinataires). La Confédération accorde plus de place à de petits pays particulièrement défavorisés. (Au début de la section 2 , nous avons cependant constaté que l'aide publique ne représentait qu'une petite partie des flux financiers suisses destinés aux pays en développement. Les capitaux privés sont investis là où semblent s'ouvrir les meilleures perspectives de rendement.) La structure des prestations du CAD reflète largement, pour sa part, la persistance de liens coloniaux et la priorité que les grandes puissances accordent aux régions d'importance stratégique.

c) Coopération technique et aide financière bilatérales suisses : destination géographique des prestations, institutions chargées de la réalisation des projets (Tableaux 3.3.)

L'aide publique suisse représente environ un pourcent des prestations correspondantes de l'ensemble des pays membres du CAD. Pour éviter une dispersion trop forte de ses versements bilatéraux, la Confédération soutient un nombre restreint de pays (principe de la concentration de l'aide). Actuellement, dix-sept pays reçoivent plus de la moitié des prestations de coopération technique et d'aide financière bilatérales suisses. Hormis I'Inde, le Bangladesh, le Pakistan et I'Indonésie, ces pays n'ont pas des populations très importantes.

La Direction de la coopération au développement et de l'aide humanitaire (DDA) assure elle-même l'exécution de plus d'un tiers des projets qu'elle soutient dans les domaines de la coopération technique et de l'aide financière. En 1983, la DDA a réalisé directement $37 \%$ des projets et elle y a consacré $44 \%$ du montant total des versements. Elle collabore simultanément avec un grand nombre d'institutions publiques et privées. Certains projets sont réalisés sous mandat par des œuvres d'entraide, des collectivités publiques, des institutions universitaires ou des firmes (des bureaux de consultants notamment) suisses. La DDA soutient activement des actions entreprises par les institutions bénévoles (voir aussi tableau 3.6.). Enfin, elle contribue de plus en plus à la réalisation de projets gérés par des organisations internationales et elle appuie les travaux d'instituts de recherche internationaux, en particulier dans le domaine de l'agriculture. 


\section{d) Répartition par secteurs économiques des prestations de coopération technique (Tableau 3.4.)}

Les principaux secteurs sont l'agriculture, l'éducation, la santé, I'hygiène et les services sociaux. A la lecture des chiffres, la part de l'agriculture $129 \%$ en 1982 et $34 \%$ en 1983) peut paraître faible compte tenu de la prépondérance de la population rurale dans les pays destinataires de la coopération technique suisse. En réalité, I'agriculture bénéficie aussi, de façon indirecte, des prestations accordées à d'autres secteurs. En outre, la majorité des projets "intégrés", qui recouvrent plusieurs secteurs, sont des projets de développement rural à forte composante agricole.

\section{e) L'aide multilatérale de la Suisse (Tableau 3.5.)}

Les prestations multilatérales consistent en contributions de caractère général aux organisations internationales. Ces versements sont souvent destinés à des programmes de développement, mais non à des pays particuliers ou à des projets spécifiquement choisis et déterminés (comme ceux qui figurent au tableau 3.3.D.).

Plus de la moitié de l'aide multilatérale suisse est versée aux organisations des Nations Unies, en particulier au Programme des Nations Unies pour le développement (PNUD).

La Confédération soutient aussi les banques régionales de développement (Afrique, Amérique et Asie) et leurs fonds spéciaux, le Fonds international pour le développement agricole (FIDA), le Comité international de la CroixRouge (CICR), etc.

Depuis 1982, la Confédération participe au financement de plusieurs fonds internationaux de développement par l'émission de "notes" (billets à ordre non négociables). Les bénéficiaires les encaissent selon un échéancier préétabli (les versements peuvent parfois s'étaler sur près d'une décennie). Les premiers encaissements ont eu lieu en 1983 pour environ 4 millions de francs.

\section{f) Dons des institutions privées bénévoles suisses} (Tableau 3.6.)

Les prestations des institutions privées bénévoles représentent une catégorie de flux financiers distincts de I'aide publique au développement. Les objectifs de ces organisations concordent cependant dans une large mesure avec les buts poursuivis par les pouvoirs publics.

La DDA et les institutions bénévoles collaborent dans la réalisation de nom- 
breux projets. Les principaux modes de collaboration sont l'exécution de projets pour le compte de la Confédération et la participation de celle-ci à des actions entreprises par les institutions privées. (Voir aussi tableau 3.3.C.)

Les dons des institutions privées bénévoles se sont élevés à 108,6 millions de francs en 1983 (1). Trois cinquièmes de cette somme provenaient des œuvres $d$ 'entraide et un tiers des œuvres missionnaires. Quatre cinquièmes ont été affectés à des projets de coopération technique et un cinquième à des actions humanitaires.

Les institutions privées bénévoles sont nombreuses mais les plus importantes effectuent la plus grande partie des dons. En 1983, vingt-sept organismes qui ont versé chacun au moins un million de francs ont assuré plus de $80 \%$ de l'ensemble des prestations privées bénévoles (2).

\section{REMARQUES}

\section{a) Interprétation des chiffres concernant l'aide publique au développement}

Les sources étant les mêmes, les remarques faites dans la section précédente à propos des statistiques du CAD s'appliquent généralement aussi ici. II convient de tenir compte également des éléments suivants :

- Pour des raisons évidentes, les statistiques de l'aide publique au développement sont bien plus précises et plus détaillées que celles des flux de capitaux privés. Cependant, il faut se rappeler que l'aide publique, en tout cas celle de la Suisse, occupe une place modeste dans l'ensemble des flux financiers à destination des pays en développement.

- Considérée en elle-même, l'aide publique apparaît comme une prestation unilatérale des donateurs. N'oublions pas la contribution du Tiers Monde au développement des économies industrialisées! Sous cet angle, l'aide suisse paraît particulièrement chiche.

1. Les chiffres concernant les dons des institutions privées bénévoles proviennent d'une enquête publiée chaque année par Swissaid. Le chiffre de 108,6 millions de francs diverge légèrement de la statistique officielle des flux financiers destinés aux pays en développement $(100,7$ millions représentant 0,05\% du PNB de la Suisse en 1983). Il comprend en effet les contributions des cantons et des communes qui passent par le canal des institutions privées bénévoles.

2. Dons supérieurs à 1 million : vingt-sept institutions et $82 \%$ des versements. Dons supérieurs à 2 millions : treize institutions et $63 \%$ des versements. Dons supérieurs à 5 millions : six institutions et $42 \%$ des versements. 
- La différence entre les versements nets et les transferts nets est moins importante que dans le cas des mouvements de capitaux privés (voir remarques concernant l'interprétation des chiffres dans la section 2, "Flux financiers"). Les paiements d'intérêts (que le CAD ne déduit pas des versements bruts) pour des prêts accordés dans le cadre de l'aide publique sont en effet modestes. En ce qui concerne la Suisse, son aide est principalement versée sous la forme de dons.

- Depuis 1979, le CAD inclut les coûts administratifs liés à la réalisation des programmes de développement dans la statistique de l'aide publique. Ces charges sont aussi comprises dans nos tableaux. Les frais administratifs encourus en Suisse correspondent actuellement à environ 2,5\% de l'aide publique au développement (voir tableau 3.1.B.).

- L'aide au développement sert dans une large mesure, même lorsqu'elle n'est pas liée, à financer l'acquisition de biens et de services dans les pays industrialisés. Grand exportateur de biens et de services, mais petit donateur, la Suisse bénéficie indirectement des prestations des autres pays développés.

\section{b) Classification des pays}

Comme pour les flux financiers (section 2), la classification des pays correspond également aux normes du CAD. Elle diffère sensiblement de la classification que I'ONU a retenue pour ses statistiques du commerce international. Le monde en développement défini par le CAD est beaucoup plus grand. II comprend aussi des pays d'Europe méridionale (Portugal, Gibraltar, Malte, Yougoslavie, Grèce, Chypre et Turquie) et deux pays socialistes d'Asie (la Chine et le Vietnam). Dans les documents du CAD, I'Espagne ne figure désormais plus parmi les pays en développement. II faut tenir compte de ces différences de classification dans les comparaisons entre les statistiques du commerce (section 1), celles des flux financiers et celles de l'aide publique au développement (sections 2 et 3 ). Pour plus de précisions, voir appendice, point 4.2.

\section{c) Taux de change}

Francs suisses pour 1 dollar :

$1981: 1,9637$

$1982: 2,0303$

$1983: 2,0902$ 


\subsection{APERÇU GENERAL DE L'AIDE PUBLIQUE AU DEVELOPPEMENT}

A. TYPES DE CONTRIBUTIONS $(1981,1982$ et 1983)

1. Versements nets déclarés par la Suisse

\begin{tabular}{|l|r|r|r||r|r|r|}
\hline \multirow{2}{*}{ Types de contributions } & \multicolumn{1}{|c||}{1981} & 1982 & \multicolumn{2}{|c||}{1983} & 1981 & \multicolumn{2}{c|}{1982} & 1983 \\
\cline { 2 - 7 } & \multicolumn{3}{|c||}{ En millions de francs } & \multicolumn{3}{c|}{ En \% du total } \\
\hline & \multicolumn{2}{|c|}{} & & & & \\
AIDE BILATERALE & 304,5 & 346,8 & 424,6 & 65,5 & 70,8 & 73,9 \\
Dons & 268,6 & 332,9 & 391,7 & 57,8 & 68,0 & 68,2 \\
Prêts & 35,9 & 13,9 & 32,9 & 7,7 & 2,8 & 5,7 \\
AIDE & & & & & & \\
MULTILATERALE & 160,6 & 142,8 & 149,6 & 34,5 & 29,2 & 26,1 \\
Dons & 97,3 & 116,0 & 132,2 & 20,9 & 23,7 & 23,0 \\
Souscriptions, etc. & 52,3 & 19,8 & 11,9 & 11,2 & 4,0 & 2,1 \\
Prêts & 11,0 & 7,0 & 5,5 & 2,4 & 1,4 & 1,0 \\
TOTAL & 465,1 & 489,6 & 574,3 & 100,0 & 100,0 & 100,0 \\
\hline
\end{tabular}

Notes :

- L'aide bilatérale comprend les contributions que la Confédération accorde aux pays bénéficiaires, directement ou par l'intermédiaire des œuvres suisses d'entraide, d'institutions universitaires, d'entreprises privées (surtout pour des projets à caractère très technique) et, lorsque les versements sont affectés à des projets spécifiques, d'organisations internationales.

- L'aide multilatérale consiste en contributions de caractère général aux organisations internationales. Ces versements sont destinés à des programmes de développement, mais non à des pays particuliers ou à des projets spécifiquement choisis et déterminés. Les souscriptions sont des participations au capital des banques régionales de développement et des contributions à leurs fonds spéciaux.

- Les coûts administratifs liés à la réalisation des programmes de développement sont comptés avec les dons bilatéraux, pour toutes les années indiquées ci-dessus, conformément aux normes actuelles du Comité d'aide au développement de l'OCDE.

- En revanche, les dons faits aux organisations privées internationales (principalement au Comité international de la Croix Rouge) sont compris ici dans l'aide multilatérale (et non bilatérale). Ces dons se sont élevés à 15,9 millions de francs en 1981, 26,3 millions en 1982 et à 33,4 millions en 1983.

- Les "notes" émises en 1982 et en 1983 en faveur de plusieurs organisations internationales (voir précisions sous les tableaux 2.1.A. et 3.5.), mais non encore encaissées, ne sont pas comptées ici. Elles représentaient environ 22 millions de francs d'aide multilatérale en 1982 et 97 millions de francs en 1983.

Sources :

DDA, Recueil de statistiques, août 1984, et informations communiquées en octobre 1984 . 


\subsection{APERCQU GENERAL DE L'AIDE PUBLIQUE AU DEVELOPPEMENT (suite)}

A. TYPES DE CONTRIBUTIONS (1981, 1982 et 1983)

2. Versements nets déclarés par I'ensemble des pays membres du CAD

\begin{tabular}{|l|r|r|r||r|r|r|}
\hline \multirow{2}{*}{$\begin{array}{c}\text { Types de } \\
\text { Contributions }\end{array}$} & 1981 & 1982 & $1983 p$ & 1981 & 1982 & $1983 p$ \\
\cline { 2 - 7 } & \multicolumn{3}{|c||}{ En millions de dollars } & \multicolumn{3}{c|}{ En \% du total } \\
\hline & 18.195 & 18.433 & 18.528 & 71,2 & 66,5 & 67,5 \\
AIDE BILATERALE & 13.180 & 13.411 & 14.126 & 51,6 & 48,4 & 51,4 \\
Dons & 5.015 & 5.022 & 4.403 & 19,6 & 18,1 & 16,0 \\
Préts & & & & & & \\
AIDE & 7.345 & 9.297 & 8.930 & 28,8 & 33,5 & 32,5 \\
MULTILATERALE & 4.024 & 4.120 & 3.961 & 15,8 & 14,9 & 14,4 \\
Dons & 3.289 & 5.175 & 4.965 & 12,9 & 18,7 & 18,1 \\
Souscriptions, etc. & 32 & 4 & 4 & 0,1 & 0,0 & 0,0 \\
Prêts & 25.540 & 27.731 & 27.458 & 100,0 & 100,0 & 100,0 \\
TOTAL & & & & & & \\
\hline
\end{tabular}

\section{Note :}

La définition des rubriques est en règle générale la même que celle du tableau précédent (classification établie par le Comité d'aide au développement de l'OCDE). Une exception y est signalée (cf. 4ème note).

\section{Sources :}

OCDE, Coopération pour le développement, Examen 1983, Annexe statistique et informations communiquées en octobre 1984. 


\subsection{APERÇU GENERAL DE L'AIDE PUBLIQUE AU DEVELOPPEMENT (suite)}

B. PRINCIPALES CATEGORIES DE L'AIDE PUBLIQUE SUISSE (1981, 1982 et 1983)

\begin{tabular}{|l|r|r|r|}
\hline \multirow{2}{*}{ Catégories } & 1981 & 1982 & 1983 \\
\hline \multirow{2}{*}{ CONFEDERATION } & \multicolumn{3}{|c|}{ (en millions de francs) } \\
\cline { 2 - 4 } Coopération technique & $\mathbf{4 5 8 , 9}$ & $\mathbf{4 8 2 , 1}$ & $\mathbf{5 6 5 , 9}$ \\
Aide financière & 193,3 & 225,7 & $\mathbf{2 5 9 , 5}$ \\
Mesures de politique & 94,2 & 90,0 & 94,9 \\
économique et commerciale & 45,3 & 35,5 & 58,7 \\
Autres mesures économiques (1) & 25,0 & - & 10,0 \\
Aide alimentaire & 44,8 & 48,7 & 55,0 \\
Aide humanitaire & 45,2 & 60,7 & 68,3 \\
Autres contributions (2) & 3,8 & 11,7 & 8,8 \\
Coûts administratifs & 12,4 & 13,3 & 14,3 \\
Remboursements de prêts & $-5,1$ & $-3,5$ & $-3,6$ \\
antérieurs & $\mathbf{6 , 1}$ & $\mathbf{7 , 5}$ & $\mathbf{8 , 4}$ \\
CANTONS ET commuNES & 5,2 & 5,9 & 6,7 \\
Coopération technique & 0,9 & 1,6 & 1,7 \\
Aide humanitaire & $\mathbf{4 6 5 , 1}$ & $\mathbf{4 8 9 , 6}$ & $\mathbf{5 7 4 , 3}$ \\
\hline TOTAL & &
\end{tabular}

1. Crédit de 35 millions de francs accordé à la Turquie dans le cadre d'un programme d'assainissement de l'économie approuvé par I'OCDE, le FMI et la Banque mondiale.

2. Part des contributions au budget régulier des Agences des Nations Unies considérée comme aide publique au développement.

Source : La DDA en 1983, tableau 1. 


\subsection{AIDE PUBLIQUE AU DEVELOPPEMENT BILATERALE}

A. REPARTITION PAR REGIONS DE DESTINATION (1983)

Comparaison entre les versements nets de la Suisse

et ceux de l'ensemble des pays membres du CAD

\begin{tabular}{|c|c|c|c|c|}
\hline \multirow{2}{*}{ Régions (1) } & \multicolumn{2}{|c|}{ Versements suisses } & \multicolumn{2}{|c|}{ Versements CAD } \\
\hline & Mio Fr. & $\%$ & Mio Fr. & $\%$ \\
\hline $\begin{array}{l}\text { AFRIQUE } \\
\text { Nord du Sahara } \\
\text { Sud du Sahara } \\
\text { Non spécifié (2) }\end{array}$ & $\begin{array}{r}191,2 \\
7,5 \\
157,1 \\
26,6\end{array}$ & $\begin{array}{r}45,0 \\
1,8 \\
37,0 \\
6,3\end{array}$ & $\begin{array}{r}14.306 \\
3.587 \\
10.425 \\
293\end{array}$ & $\begin{array}{r}36,8 \\
9,2 \\
26,8 \\
0,8\end{array}$ \\
\hline $\begin{array}{l}\text { AMERIQUE } \\
\text { Centre (avec Caraïbes) } \\
\text { Sud } \\
\text { Non spécifié (2) }\end{array}$ & $\begin{array}{l}64,0 \\
29,8 \\
23,2 \\
11,1\end{array}$ & $\begin{array}{r}15,1 \\
7,0 \\
5,5 \\
2,6\end{array}$ & $\begin{array}{r}5.385 \\
3.653 \\
1.512 \\
219\end{array}$ & $\begin{array}{r}13,8 \\
9,4 \\
3,9 \\
0,6\end{array}$ \\
\hline $\begin{array}{l}\text { ASIE } \\
\text { Moyen-Orient } \\
\text { Asie du Sud } \\
\text { Extrême-Orient } \\
\text { Non spécifié (2) }\end{array}$ & $\begin{array}{r}122,1 \\
11,2 \\
91,5 \\
14,4 \\
5,0\end{array}$ & $\begin{array}{r}28,8 \\
2,6 \\
21,6 \\
3,4 \\
1,2\end{array}$ & $\begin{array}{r}12.910 \\
3.667 \\
4.699 \\
4.375 \\
169\end{array}$ & $\begin{array}{r}33,2 \\
9,4 \\
12,1 \\
11,2 \\
0,4\end{array}$ \\
\hline OCEANIE & 0,3 & 0,1 & 1.940 & 5,0 \\
\hline EUROPE & 9,0 & 2,1 & 810 & 2,1 \\
\hline NON SPECIFIE (2) & 38,0 & 8,9 & 3.543 & 9,1 \\
\hline TOTAL & 424,6 & 100,0 & 38.894 & 100,0 \\
\hline
\end{tabular}

1. Pour les explications concernant la classification, voir point 4.2. "Composition des groupes de pays".

2. Ces prestations ne sont pas spécifiées géographiquement (totalement ou en partie) car elles concernent plusieurs régions simultanément. II s'agit de contributions à des cours, à des programmes, de crédits globaux pour les boursiers et pour le financement des recherches, du soutien de programmes entrepris par les œuvres d'entraide ou encore des prestations cantonales et communales. Les frais administratifs liés à la gestion de l'aide ne sont pas non plus ventilés géographiquement.

Note :

L'aide publique au développement bilatérale comprend I'ensemble des volets bilatéraux de la coopération technique, de l'aide financière, des mesures de politique économique et commerciale, ainsi que des aides alimentaire et humanitaire.

\section{Sources :}

- La DDA en 1983, Annexe II.

- OCDE, Répartition géographique des ressources financières mises à la disposition des pays en développement. Chiffres provisoires. 


\subsection{AIDE PUBLIQUE AU DEVELOPPEMENT BILATERALE (suite)}

B. LES VINGT PREMIERS PAYS DESTINATAIRES (1983)

Comparaison entre les versements nets de la Suisse et ceux de l'ensemble des pays membres du CAD

\begin{tabular}{|c|c|c|c|c|c|c|c|}
\hline \multicolumn{4}{|c|}{ VERSEMENTS DE LA SUISSE } & \multicolumn{4}{|c|}{$\begin{array}{l}\text { VERSEMENTS DES PAYS MEMBRES DU } \\
\text { COMITE D'AIDE AU DEVELOPPEMENT }\end{array}$} \\
\hline Pays & Mio Fr. & $\%$ & $\Sigma \%$ & Pays & Mio Fr. & $\%$ & $\Sigma \%$ \\
\hline 1. Inde & 45,9 & 10,8 & 10,8 & 1. Israël & 2.823 & 7,3 & 7,3 \\
\hline 2. Népal & 22,5 & 5,3 & 16,1 & 2. Egypte & 2.589 & 6.7 & 13,9 \\
\hline 3. Madagascar & 22,1 & 5,2 & 21,3 & 3. Inde & 1.522 & 3,9 & 17,8 \\
\hline 4. Rwanda & 15,5 & 3,7 & 25,0 & 4. Indonésie & 1.299 & 3,3 & 21,2 \\
\hline 5. Mali & 15,3 & 3,6 & 28,6 & 5. Bangladesh & 1.223 & 3,1 & 24,3 \\
\hline 6. Soudan & 12,7 & 3,0 & 31,6 & 6. Chine & 1.020 & 2.6 & 26,9 \\
\hline 7. Tanzanie & 12,2 & 2,9 & 34,4 & 7. Soudan & 928 & 2,4 & 29,3 \\
\hline 8. Honduras & 10,9 & 2,6 & 37,0 & 8. Tanzanie & 916 & 2,4 & 31,7 \\
\hline 9. Sri Lanka & 9,4 & 2,2 & 39,2 & 9. Réunion & 809 & 2,1 & 33,8 \\
\hline 10. Pérou & 9,4 & 2,2 & 41,4 & 10. Philippines & 742 & 1,9 & 35,7 \\
\hline 11. Indonésie & 8.7 & 2,1 & 43,5 & 11. Sri Lanka & 719 & 1,8 & 37,5 \\
\hline 12. Turquie & 8,7 & 2,0 & 45,5 & 12. Thailande & 718 & 1,8 & 39,4 \\
\hline 13. Niger & 7,8 & 1,8 & 47,4 & 13. Kenya & 707 & 1,8 & 41,2 \\
\hline 14. Sénégal & 7,5 & 1,8 & 49,1 & 14. Turquie & 616 & 1,6 & 42,8 \\
\hline 15. Bangladesh & 6,8 & 1,6 & 50,7 & 15. Papouasie - N. Guinée & e 576 & 1,5 & 44,3 \\
\hline 16. Bourkina Faso & 6,1 & 1,4 & 52,2 & 16. Martinique & 567 & 1,5 & 45,7 \\
\hline 17. Zimbabwe & 6,1 & 1,4 & 53,6 & 17. Pakistan & 530 & 1,4 & 47.1 \\
\hline 18. Mozambique & 5,6 & 1,3 & 54,9 & 18. El Salvador & 491 & 1,3 & 48,3 \\
\hline 19. Yémen & 5,4 & 1,3 & 56,2 & 19. Pérou & 482 & 1,2 & 49,6 \\
\hline 20. Cameroun & 5,4 & 1,3 & 57,4 & 20. Costa Rica & 460 & 1,2 & 50,8 \\
\hline Autres pays & 86,7 & 20,4 . & 77,9 & Autres pays & 14.162 & 36,4 & 87,2 \\
\hline Non spécifié (1) & 93,9 & 22,1 & 100,0 & Non spécifié (1) & 4.992 & 12,8 & 100,0 \\
\hline Total & 424,6 & 100,0 & 100,0 & Total & 38.894 & 100,0 & 100,0 \\
\hline
\end{tabular}

1. Ces prestations ne sont pas spécifiées car elles concernent plusieurs pays simultanément. (Voir précisions données sous le tableau précédent, note 2.)

Sources :

- La DDA en 1983, Annexe II.

- OCDE, Répartition géographique des ressources financières mises à la disposition des pays en développement. Chiffes provisoires. 


\subsection{COOPERATION TECHNIQUE ET AIDE FINANCIERE BILATERALES SUISSES}

A. REPARTITION PAR REGIONS ET PAR PAYS DE CONCENTRATION (1982 et 1983)

Versements nets de la DDA

\begin{tabular}{|c|c|c|c|c|}
\hline \multirow{2}{*}{$\begin{array}{c}\text { Région } \\
\text { Pays de concentration }\end{array}$} & \multicolumn{2}{|c|}{1981} & \multicolumn{2}{|c|}{$1982 p$} \\
\hline & Mio Fr. & $\%$ & Mio Fr. & $\%$ \\
\hline $\begin{array}{l}\text { AFRIQUE } \\
\text { Rwanda } \\
\text { Mali } \\
\text { Tanzanie } \\
\text { Madagascar } \\
\text { Kenya } \\
\text { Niger } \\
\text { Bourkina Faso (Haute-Volta) } \\
\text { Mozambique } \\
\text { Bénin } \\
\text { Zone du Sahel } \\
\text { Autres pays (1) }\end{array}$ & $\begin{array}{r}105,8 \\
18,3 \\
12,6 \\
9,9 \\
8,2 \\
6,8 \\
5,0 \\
2,7 \\
2,3 \\
1,6 \\
6,2 \\
32,2\end{array}$ & 41,8 & $\begin{array}{r}118,1 \\
14,7 \\
14,4 \\
11,1 \\
6,3 \\
4,5 \\
7,0 \\
5,1 \\
4,7 \\
3,8 \\
5,7 \\
40,8\end{array}$ & 41,1 \\
\hline $\begin{array}{l}\text { AMERIQUE LATINE } \\
\text { Honduras } \\
\text { Pérou } \\
\text { Bolivie } \\
\text { Autres pays (1) }\end{array}$ & $\begin{array}{r}29,2 \\
6,3 \\
6,0 \\
1,5 \\
15,4\end{array}$ & 11,5 & $\begin{array}{r}52,0 \\
6,1 \\
5,7 \\
3,7 \\
32,7\end{array}$ & 18,1 \\
\hline $\begin{array}{l}\text { ASIE ET OCEANIE } \\
\text { Inde } \\
\text { Népal } \\
\text { Bangladesh } \\
\text { Indonésie } \\
\text { Pakistan } \\
\text { Autres pays (1) }\end{array}$ & $\begin{array}{r}91,5 \\
34,5 \\
22,2 \\
8,2 \\
6,6 \\
5,4 \\
14,6\end{array}$ & 36,2 & $\begin{array}{r}95,3 \\
42,4 \\
22,1 \\
5,5 \\
7,6 \\
2,8 \\
14,9\end{array}$ & 33,2 \\
\hline EUROPE & 2,9 & 1,1 & 1,0 & 0,3 \\
\hline NON CLASSE (2) & 23,6 & 9,3 & 20,8 & 7,3 \\
\hline TOTAL & 253,0 & 100,0 & 287,2 & 100,0 \\
\hline
\end{tabular}

1. Une partie importante de ces versements concerne des projets régionaux.

2. Comprend des versements à des institutions suisses pour des programmes de formation et de coopération au développement, des bourses d'études, etc.

Source : DDA, Rapport annuel 1982, p. 8 et 1983, p. 6. 


\subsection{COOPERATION TECHNIQUE ET AIDE FINANCIERE BILATERALES SUISSES (suite)}

\section{B. REPARTITION SELON LES MODALITES D’EXECUTION DES PROJETS (1983)}

Nombre d'actions en cours et versements nets de la DDA

\begin{tabular}{|c|c|c|c|c|c|c|c|}
\hline \multirow{2}{*}{ Modalités d'exécution } & \multicolumn{2}{|c|}{$\begin{array}{l}\text { Coopération } \\
\text { technique }\end{array}$} & \multicolumn{2}{|c|}{$\begin{array}{c}\text { Aide } \\
\text { financière }\end{array}$} & \multicolumn{3}{|c|}{ Total } \\
\hline & Nombre & $\begin{array}{c}\text { Mio } \\
\text { Fr. }\end{array}$ & Nombre & $\begin{array}{c}\text { Mio } \\
\text { Fr. }\end{array}$ & Nombre & $\begin{array}{c}\text { Mio } \\
\text { Fr. }\end{array}$ & $\%$ \\
\hline $\begin{array}{l}\text { Actions réalisées directe- } \\
\text { ment par la DDA }\end{array}$ & 158 & 69,6 & 31 & 56,5 & 189 & 126,1 & 43,9 \\
\hline $\begin{array}{l}\text { Actions réalisées en régie } \\
\text { par des institutions suisses }\end{array}$ & 91 & 53,5 & 1 & 3,0 & 92 & 56,5 & 19,7 \\
\hline $\begin{array}{l}\text { Contributions à des insti- } \\
\text { tutions suisses pour des } \\
\text { projets spécifiques }\end{array}$ & 125 & 32,9 & - & - & 125 & 32,9 & 11,4 \\
\hline $\begin{array}{l}\text { Contributions à des orga- } \\
\text { nisations internationales } \\
\text { pour des projets } \\
\text { spécifiques }\end{array}$ & 90 & 51,5 & 18 & 20,2 & 108 & 71,7 & 25,0 \\
\hline TOTAL & 464 & 207,5 & 50 & 79,7 & 514 & 287,2 & 100,0 \\
\hline
\end{tabular}

Notes :

La collaboration entre la DDA et les institutions suisses revêt généralement l'une des deux formes suivantes :

- Exécution de projets en régie : la DDA fixe, d'entente avec ses partenaires dans les pays en développement, les buts des projets et les moyens à mettre en œuvre. Le rôle des régisseurs est défini dans les contrats de régie : il peut se limiter à des aspects techniques des projets ou s'étendre à l'engagement de personnel et à l'achat de matériel. La DDA conserve la responsabilité principale de l'action. Le régisseur est une institution bénévole (voir tableau suivant), une collectivité publique, une institution universitaire ou une entreprise privée (firme de consultants par exemple).

- Contributions financières pour des projets spécifiques : il s'agit surtout d'actions entreprises par les institutions bénévoles. La DDA décide de soutenir des projets dont elle a approuvé les objectifs; elle contrôle l'exécution et les comptes. La DDA appuie aussi des activités de formation professionnelle poursuivies par des collectivités publiques et des institutions universitaires.

Les modalités de la collaboration entre la DDA et les organisations internationales pour des projets spécifiques sont indiquées sous le tableau 3.3.D.

\section{Sources :}

DDA, Rapport annuel 1983, p. 8 et La DDA en 1983, Tableaux 9 et 10. 


\subsection{COOPERATION TECHNIQUE ET AIDE FINANCIERE BILATERALES SUISSES (suite)}

\section{CONTRIBUTIONS AUX ACTIVITES DE COOPERATION TECHNIQUE D'INSTITUTIONS PRIVEES BENEVOLES SUISSES (1983)}

\section{Versements nets de la DDA}

\begin{tabular}{|c|c|c|c|}
\hline \multirow[t]{2}{*}{ Institutions } & $\begin{array}{l}\text { Actions des } \\
\text { institutions }\end{array}$ & $\begin{array}{c}\text { Actions en } \\
\text { régie }\end{array}$ & Total \\
\hline & Mio $\mathrm{Fr}$. & Mio $\mathrm{Fr}$. & Mio Fr. \\
\hline Intercoopération & - & 23,0 & 23,0 \\
\hline Helvetas & 9,1 & 9,7 & 18,8 \\
\hline Swisscontact & 2,2 & 3,3 & 5,5 \\
\hline $\begin{array}{l}\text { Organisation } \\
\text { Reconstruction Travail (ORT) }\end{array}$ & - & 3,2 & 3,2 \\
\hline Swissaid & 2,6 & - & 2,6 \\
\hline Action de Carême & 1,6 & - & 1,6 \\
\hline Enfants du Monde & - & 1.5 & 1,5 \\
\hline Caritas & 1.3 & - & 1,3 \\
\hline $\begin{array}{l}\text { Institut panafricain pour le } \\
\text { développement (IPD) }\end{array}$ & 1,0 & - & 1,0 \\
\hline Croix-Rouge Suisse (CRS) & 0,2 & 0,8 & 1,0 \\
\hline $\begin{array}{l}\text { Association "Se servir de la saison } \\
\text { sèche en savane et au Sahel" (6S) }\end{array}$ & 0,9 & - & 0.9 \\
\hline Pain pour le Prochain & 0,8 & - & 0,8 \\
\hline Entraide protestante suisse (EPER) & 0,1 & 0,6 & 0,7 \\
\hline $\begin{array}{l}\text { Mouvement chrétien } \\
\text { pour la paix (MCP) }\end{array}$ & 0,7 & - & 0.7 \\
\hline $\begin{array}{l}\text { Association suisse des } \\
\text { médecins missionnaires catholiques }\end{array}$ & - & 0,6 & 0,6 \\
\hline $\begin{array}{l}\text { Centre suisse pour la technologie } \\
\text { appropriée (SKAT) }\end{array}$ & 0,5 & - & 0,5 \\
\hline $\begin{array}{l}\text { Cuvre suisse } \\
\text { d'entraide ouvrière (OSEO) }\end{array}$ & 0,4 & - & 0,4 \\
\hline Schweiz. Ostasien Mission (SOMA) & 0,3 & - & 0,3 \\
\hline Association suisse des amis d'Haïti & 0,3 & - & 0,3 \\
\hline Société missionnaire de Béthlehem & 0,3 & - & 0,3 \\
\hline Schwestern vom Heiligen Kreuz & 0,2 & - & 0,2 \\
\hline $\begin{array}{l}\text { Centre de coordination, de documen- } \\
\text { tation et d'information pour la } \\
\text { formation professionnelle dans les } \\
\text { pays en voie de développement } \\
\text { (KODIS) }\end{array}$ & 0,2 & - & 0,2 \\
\hline Institut d'action culturelle (IDAC) & - & 0,2 & 0,2 \\
\hline Unité & 0,1 & - & 0,1 \\
\hline $\begin{array}{l}\text { Schweiz. Missionsprokura } \\
\text { der Kapuziner }\end{array}$ & 0,1 & - & 0,1 \\
\hline $\begin{array}{l}\text { Commission tiers monde des } \\
\text { unions chrétiennes féminines }\end{array}$ & 0,1 & - & 0,1 \\
\hline Autres institutions (1) & 4,3 & 0,3 & 4,6 \\
\hline TOTAL & 27,3 & 43,2 & 70,5 \\
\hline
\end{tabular}

1. Y compris crédits globaux pour les volontaires engagés par diverses œuvres d'entraide privées.

Note :

Concernant la distinction faite entre les contributions financières pour des actions spécifiques des institutions et l'exécution de projets en régie, voir notes du tableau précédent

Source :

La DDA en 1983, Tableau 11 


\subsection{COOPERATION TECHNIQUE ET AIDE FINANCIERE BILATERALES SUISSES (fin)}

D. CONTRIBUTIONS A DES ORGANISATIONS INTERNATIONALES (POUR DES PROJETS SPECIFIQUES) ET A DES INSTITUTS DE RECHERCHE (1983)

Versements nets de la DDA

\begin{tabular}{|c|c|c|c|c|}
\hline & $\begin{array}{c}\text { Aide } \\
\text { associée } \\
\text { Mio Fr. }\end{array}$ & $\begin{array}{l}\text { Cofinan- } \\
\text { cement } \\
\text { Mio Fr. }\end{array}$ & $\begin{array}{l}\text { Autres } \\
\text { contrib. } \\
\text { Mio Fr. }\end{array}$ & $\begin{array}{l}\text { Total } \\
\text { Mio Fr. }\end{array}$ \\
\hline Organisations des Nations Unies (N.U) & 26,2 & - & - & 26,2 \\
\hline $\begin{array}{l}\text { Organisation des N.U. pour l'alimentation } \\
\text { et l'agriculture (FAO) }\end{array}$ & 9,8 & - & - & 9,8 \\
\hline Fonds des N.U. pour I'enfance (UNICEF) & 8,3 & - & - & 8,3 \\
\hline Organisation internationale du travail (OIT) & 3,0 & - & - & 3,0 \\
\hline $\begin{array}{l}\text { Union internationale } \\
\text { des télécommunications (UIT) }\end{array}$ & 0,7 & - & - & 0,7 \\
\hline $\begin{array}{l}\text { Organisation des N.U. pour l'éducation, } \\
\text { la science et la culture (UNESCO) }\end{array}$ & 0,6 & - & - & 0,6 \\
\hline Organisation mondiale de la santé (OMS) & 0,5 & - & - & 0,5 \\
\hline Autres organisations & 3,3 & - & - & 3,3 \\
\hline Institutions financières de développement & - & 20,2 & - & 20,2 \\
\hline $\begin{array}{l}\text { Association internationale de } \\
\text { développement (IDA) }\end{array}$ & - & 12,2 & - & 12,2 \\
\hline $\begin{array}{l}\text { Banque interaméricaine de } \\
\text { développement (BID) }\end{array}$ & - & 6,0 & - & 6,0 \\
\hline Banque asiatique de développement ( $A D B$ ) & - & 2,0 & - & 2,0 \\
\hline Autres organisations internationales & 0,8 & - & - & 0,8 \\
\hline Instituts de recherche internationaux & - & - & 24,6 & 24,6 \\
\hline $\begin{array}{l}\text { Centres de recherche du Groupe consultatif } \\
\text { pour la recherche agricole internationale } \\
\text { (CG|AR) }\end{array}$ & - & - & 18,3 & 18,3 \\
\hline Autres instituts de recherche & - & - & 6,3 & 6,3 \\
\hline TOTAL & 27,0 & 20,2 & 24,6 & 71,8 \\
\hline
\end{tabular}

\section{Notes :}

Depuis 1979, les contributions accordées à des institutions internationales pour la réalisation de projets spécifiquement désignés par les pays donateurs sont comptées au nombre des prestations bilatérales. (Les notifications au CAD se font sur cette base depuis 1980.)

On entend par aide associée le financement d'un projet administré par une organisation internationale, mais choisi et suivi par le bailleur de fonds. Par cofinancement, on entend l'intervention simultanée de plusieurs fournisseurs d'aide (bilatéraux ou multilatéraux) extérieurs au pays bénéficiaire, dans le financement d'un projet.

L'appui de la DDA aux travaux d'instituts de recherche internationaux comprend des contributions ordinaires et le soutien de projets ou de programmes particuliers. II s'agit principalement de recherches agricoles.

Sources :

La DDA en 1983, tableaux 13 et 14. 
3.4. COOPERATION TECHNIQUE BILATERALE SUISSE (1982 et 1983) Répartition par secteurs économiques (Versements nets)

\begin{tabular}{|c|c|c|c|c|}
\hline \multirow{2}{*}{ Secteurs économiques } & \multicolumn{2}{|c|}{1982} & \multicolumn{2}{|c|}{1983} \\
\hline & Mio $\mathrm{Fr}$ & r. $\%$ & Mio $\mathrm{Fr}$ & $\%$ \\
\hline $\begin{array}{l}\text { AGRICULTURE } \\
\text { Production agricole } \\
\text { Sylviculture, arboriculture } \\
\text { Irrigation, services agricoles } \\
\text { Elevage } \\
\text { Autres }\end{array}$ & $\begin{array}{l}52,8 \\
. \cdot \\
. \\
. \\
. \\
. \\
.\end{array}$ & 28,8 & $\begin{array}{r}70,6 \\
25,8 \\
14,0 \\
7,2 \\
7,4 \\
16,2\end{array}$ & 34,0 \\
\hline $\begin{array}{l}\text { INDUSTRIE } \\
\text { Industries liées à l'agriculture } \\
\text { Autres industries manufacturières }\end{array}$ & $\begin{array}{l}17,1 \\
\cdot . \\
\cdot .\end{array}$ & 9,3 & $\begin{array}{r}18,9 \\
12,6 \\
6,3\end{array}$ & 9,1 \\
\hline $\begin{array}{l}\text { COMMUNICATIONS, TRANSPORTS, } \\
\text { CONSTRUCTION }\end{array}$ & 5,9 & 3,2 & 6,6 & 3,2 \\
\hline COMMERCE, BANQUES, TOURISME & 5,3 & 2,9 & 6,1 & 2,9 \\
\hline ADMINISTRATION PUBLIQUE & 0,3 & 0,2 & 0,3 & 0,2 \\
\hline $\begin{array}{l}\text { EDUCATION } \\
\text { Enseignement universitaire } \\
\text { Enseignement technique supérieur } \\
\text { Alphabétisation et autres formations }\end{array}$ & $\begin{array}{l}25,4 \\
\cdot \cdot \\
\cdot \cdot \\
.\end{array}$ & 13,9 & $\begin{array}{r}29,1 \\
8,4 \\
6,7 \\
14,0\end{array}$ & 14,0 \\
\hline SANTE, HYGIENE, SERVICES SOCIAUX & 18,9 & 10,3 & 23,3 & 11,2 \\
\hline MULTISECTEURS (1) & 26,3 & 14,4 & 23,7 & 11,4 \\
\hline NON CLASSE & 31,1 & 17,0 & 28,9 & 13,9 \\
\hline TOTAL & 183,1 & 100,0 & 207,5 & 100,0 \\
\hline
\end{tabular}

1. Projets régionaux de développement rural, ressources naturelles et humaines, développement communautaire, planification.

Source : DDA, informations communiquées en octobre 1984. 


\subsection{AIDE PUBLIQUE AU DEVELOPPEMENT MULTILATERALE (1981, 1982 et 1983)}

Versements nets

\begin{tabular}{|c|c|c|c|}
\hline Organisations & \multicolumn{3}{|c|}{ En millions de francs } \\
\hline 1. Organisations des Nations Unies (N.U.) & 80,6 & 88,7 & 97,8 \\
\hline Programme des N.U. pour le développement (PNUD) & 38,8 & 38,3 & 50,2 \\
\hline Programme alimentaire mondial (PAM) & 13,0 & 15,0 & 11,0 \\
\hline $\begin{array}{l}\text { Office des N.U. pour les réfugiés de Palestine } \\
\text { (UNRWA) }\end{array}$ & 8,7 & 8,7 & 9,0 \\
\hline Fonds des N.U. pour l'enfance (UNICEF) & 8,3 & 8,5 & 8,2 \\
\hline Haut Commissariat des N.U. pour les réfugiés (HCR) & 2,7 & 3,7 & 4,2 \\
\hline $\begin{array}{l}\text { Fonds des N.U. pour les activités en matière de } \\
\text { population (FNUAP) }\end{array}$ & 3,0 & 3,3 & 3,6 \\
\hline $\begin{array}{l}\text { Organisation mondiale de la santé (OMS), } \\
\text { programmes spéciaux }\end{array}$ & 1,7 & 2,6 & 2,3 \\
\hline Autres organisations & 4,4 & 8,6 & 9,3 \\
\hline $\begin{array}{l}\text { 2. Banques régionales de développement } \\
\text { et leurs fonds spéciaux }\end{array}$ & 47,2 & 19,8 & 11,9 \\
\hline Banque africaine de développement (BAD) & - & 6,8 & 6,8 \\
\hline Fonds africain de développement (FAD) & 26,9 & - & * \\
\hline Banque asiatique de développement (ADB) & 2,3 & 0,1 & - \\
\hline Fonds asiatique de développement (ADF) & 10,0 & 11,6 & * \\
\hline Fonds d'assistance technique de I'ADB & - & - & 1,0 \\
\hline Banque interaméricaine de développement (BID) & 0,7 & 1,3 & $0,5^{*}$ \\
\hline Fonds opérations spéciales de la BID & 7,3 & * & $3,6^{*}$ \\
\hline 3. Autres organismes multilatéraux & 16,9 & 8,0 & 6,5 \\
\hline $\begin{array}{l}\text { Fonds international pour le développement } \\
\text { agricole (FIDA) }\end{array}$ & - & * & * \\
\hline $\begin{array}{l}\text { Fonds monétaire international (FMI) } \\
\text { compte de bonification des intérêts }\end{array}$ & 5,2 & - & - \\
\hline $\begin{array}{l}\text { Fonds de l'Association de libre échange (AELE) } \\
\text { en faveur du Portugal }\end{array}$ & 11,0 & 7,0 & 5,5 \\
\hline Autres organismes & 0,7 & 1,0 & 1,0 \\
\hline 4. Organisations privées internationales & 15,9 & 26,3 & 33,4 \\
\hline Comité international de la Croix Rouge (CICR) & 15,9 & 26,3 & 33,4 \\
\hline TOTAL & 160,6 & 142,8 & 149,6 \\
\hline
\end{tabular}

- Plusieurs paiements ont été effectués par l'émission de "notes" (reconnaissances de dettes irrévocables). Voir explication sous le tableau 2.1.A. II s'agit des montants suivants (en millions de francs) :

- $1982: 8,0$ (FSO-BID), 14,0 (FIDA). Total : 22,0.

- $1983: 51,3$ (FAD), 19,9 (ADF), 2,2 (BID), 13,5 (FSO-BID), 14,4 (FIDA). Total : 101,3.

Les bénéficiaires des "notes" n'ont rien encaissé en 1982. En 1983, ils ont effectué les tirages suivants (en millions de francs) : 0,5 (BID), 3,6 (FSO-BID). Total : 4,1.

Source : La DDA en 1983, tableau 5 et liste des paiements effectués sous forme de "notes" en 1982 et 1983. 


\subsection{DONS DES INSTITUTIONS PRIVEES BENEVOLES SUISSES (1983)}

Versements destinés à des actions de coopération technique et d'aide humanitaire

\begin{tabular}{|c|c|c|c|c|c|c|c|c|}
\hline \multirow{3}{*}{ Institutions (1) } & & \multicolumn{3}{|c|}{ Coopération technique } & \multicolumn{3}{|c|}{ Aide humanitaire } & \multirow{2}{*}{$\begin{array}{c}\text { Total } \\
\text { des } \\
\text { dons } \\
\text { privés }\end{array}$} \\
\hline & & $\begin{array}{l}\text { Dons } \\
\text { privés }\end{array}$ & $\begin{array}{l}\text { Contrib. } \\
\text { fédérales }\end{array}$ & & $\begin{array}{l}\text { Dons } \\
\text { privés }\end{array}$ & $\begin{array}{l}\text { Contrib. } \\
\text { fédérales }\end{array}$ & Total & \\
\hline & & \multicolumn{7}{|c|}{ En millions de francs } \\
\hline Caritas & $E$ & 5,2 & 1,6 & 6,8 & 6,7 & 0,5 & 7,2 & 11,8 \\
\hline $\begin{array}{l}\text { Entraide protestante de Suisse } \\
\text { (EPER) / Hilfswerk der Evangel. } \\
\text { Kirchen der Schweiz (HEKS) }\end{array}$ & $E$ & 5,5 & 1,1 & 6,6 & 3,1 & 0,7 & 3,8 & 8,6 \\
\hline Institut Panafricain & $A$ & 7,2 & 1,2 & 8,4 & - & - & - & 7,2 \\
\hline $\begin{array}{l}\text { Action de Carême des } \\
\text { catholiques suisses / Fastenopfer } \\
\text { der Schweizer Katholiken }\end{array}$ & $E$ & 6,4 & 0,7 & 7,1 & - & - & - & 6,4 \\
\hline Missionsgesellschaft Bethlehem & M & 6,3 & 0,3 & 6,6 & - & - & - & 6,3 \\
\hline $\begin{array}{l}\text { Croix Rouge Suisse (CRS) / } \\
\text { Schweiz. Rotes Kreuz (SRK) }\end{array}$ & $\mathrm{E}$ & 2,9 & 3,0 & 5,9 & 2,1 & 5,6 & 7,7 & 5,0 \\
\hline Institut IngenbohI & M & 4,5 & 0,1 & 4,6 & 0,4 & - & 0,4 & 4,9 \\
\hline Caritas Kinderhilfe Bethlehem & $E$ & 3,6 & - & 3,6 & 0,0 & 0,1 & 0,1 & 3,6 \\
\hline Benediktiner-Mission Uznach & M & 2,9 & 0,7 & 3,6 & 0,3 & 0,1 & 0,4 & 3,2 \\
\hline Emmaüs & $\mathrm{E}$ & 3,2 & 0,1 & 3,3 & - & - & - & 3,2 \\
\hline Basler Mission & M & 2,9 & 0,6 & 3,5 & 0,3 & - & 0,3 & 3,1 \\
\hline Helvetas & $\mathrm{E}$ & 2,9 & 18,1 & 20,9 & - & - & - & 2,9 \\
\hline Institut Menzingen & M & 1,6 & - & 1,6 & 0,5 & - & 0,5 & 2,1 \\
\hline Terre des Hommes Schweiz, Basel & $\mathrm{E}$ & 1,6 & 0,0 & 1,7 & 0,3 & - & 0,3 & 2,0 \\
\hline Swisscontact & $\mathrm{E}$ & 1,9 & 3,2 & 5,1 & - & - & - & 1,9 \\
\hline Missions-Verkehrsaktion (MIVA) & $E$ & 1,9 & - & 1,9 & - & - & - & 1,9 \\
\hline Christl. Hilfswerk International & $\mathrm{E}$ & 0,5 & - & 0,5 & 1,3 & - & 1,3 & 1,8 \\
\hline Armée du Salut / Die Heilsarmee & M & 0,8 & 0,4 & 1,2 & 0,8 & - & 0,8 & 1,6 \\
\hline Swissaid & $\mathrm{E}$ & 1,6 & 2,7 & 4,2 & - & - & - & 1,6 \\
\hline Brücke der Bruderhilfe & $\mathrm{E}$ & 1,6 & - & 1,6 & - & - & - & 1,6 \\
\hline $\begin{array}{l}\text { Assoc. suisse d'aide à l'hôpital } \\
\text { A. Schweitzer }\end{array}$ & $\mathrm{E}$ & 1,4 & 0,1 & 1,5 & - & - & - & 1,4 \\
\hline Schweiz. Arbeiter Hilfswerk (SAH) & $E$ & 0,5 & 0,4 & 0,9 & 0,9 & 0,6 & 1,5 & 1,3 \\
\hline Seraphisches Liebeswerk Solothurn & $M$ & 1,1 & - & 1,1 & 0,2 & - & 0,2 & 1,3 \\
\hline Enfants du Monde & $E$ & 0,7 & 1,6 & 2,4 & 0,5 & - & 0,5 & 1,2 \\
\hline Terre des Hommes Suisse, Genève & $\mathrm{E}$ & 1,0 & - & 1,0 & 0,2 & - & 0,2 & 1,2 \\
\hline Institut Baldegg & M & 1.0 & - & 1,0 & 0,2 & - & 0,2 & 1,2 \\
\hline Frères de nos frères & $E$ & 1,0 & 0,1 & 1,1 & 0,0 & - & 0,0 & 1,0 \\
\hline Autres institutions & & 15,3 & 7,8 & 23,0 & 4,0 & 2,1 & 6,1 & 19,2 \\
\hline $\begin{array}{l}\text { TOTAL } \\
\text { dont : }\end{array}$ & & 86,8 & 43,8 & 130,6 & 21,8 & 9,7 & 31,5 & 108,6 \\
\hline Cuvres d'entraide & $E$ & 47,3 & 38,8 & 86,1 & 16,9 & 9,2 & 26,2 & 64,2 \\
\hline CEuvres missionnaires & $M$ & 29,7 & 3,5 & 33,1 & 4,8 & 0,5 & 5,3 & 34,5 \\
\hline Autres organismes & $A$ & 9,9 & 1,6 & 11,5 & 0,1 & - & 0,1 & 10,0 \\
\hline
\end{tabular}

Voir notes page suivante. 
Notes du tableau 3.6.

1. A côté des institutions, figure l'indication de leur fonction, telle qu'elle apparaît dans nos sources : œuvre d'entraide (E), œuvre missionnaire $(M)$ ou autre organisme $(A)$.

Notes :

Les dons des institutions privées bénévoles, qui représentaient 0,05\% du produit national brut de la Suisse en 1982, ne sont pas compris dans le montant de l'aide publique au développement. Les objectifs des œuvres privées concordent cependant dans une large mesure avec les buts poursuivis par les pouvoirs publics. La participation fédérale au financement des institutions bénévoles sert à soutenir leurs propres actions et à couvrir les dépenses de projets qu'elles exécutent sous mandat (voir tableau 3.3.C.).

Les versements indiqués ci-dessus correspondent aux déclarations faites par les institutions. La statistique comprend les versements effectifs pour l'année 1983 destinés à des actions de coopération technique et d'aide humanitaire, à l'exclusion des dépenses liées aux activités missionnaires au sens strict (formation des prêtres, construction d'églises, etc.).

Les institutions mentionnées dans le tableau ont effectué des dons (financés par leurs propres recettes, y compris les contributions et cantons et de communes, mais à l'exclusion des contributions fédérales) s'élevant au moins à un million de francs. Les institutions sont classées selon l'importance de ces prestations.

\section{Source :}

Swissaid, Schweizerische Hilfe für Entwicklungs/änder / Aide suisse aux pays en développement 1983, tableau III.2, "Prestations privées par organisations", pp. 59-86. 